\title{
Polynomial recursion formula for linear Hodge integrals
}

\author{
Motohico Mulase and Naizhen Zhang
}

\begin{abstract}
We establish a polynomial recursion formula for linear Hodge integrals. It is obtained as the Laplace transform of the cut-and-join equation for the simple Hurwitz numbers. We show that the recursion recovers the Witten-Kontsevich theorem when restricted to the top degree terms, and also the combinatorial factor of the $\lambda_{g}$ formula as the lowest degree terms.
\end{abstract}

1. Introduction

2. Hurwitz numbers

3. The cut-and-join equation

4. Laplace transform and the Lambert curve

5. The topological recursion as a Laplace transform

6. The Witten-Kontsevich theorem and the $\lambda_{g}$ formula

References

\section{Introduction}

The purpose of this paper is to establish a topological recursion formula for linear Hodge integrals in terms of polynomial generating functions. Let $\overline{\mathcal{M}}_{g, \ell}$ be the Deligne-Mumford moduli stack of stable curves of genus $g$ and $\ell$ distinct marked points subject to $2 g-2+\ell>0$. We denote by $\psi_{i}$ the $i$ th 
cotangent class of $\overline{\mathcal{M}}_{g, \ell}$, and by $\lambda_{j}=c_{j}(\mathbb{E})$ the $j$ th Chern class of the Hodge bundle $\mathbb{E}$ on $\overline{\mathcal{M}}_{g, \ell}$. By linear Hodge integrals we mean the rational numbers

$$
\left\langle\tau_{n_{1}} \cdots \tau_{n_{\ell}} \lambda_{j}\right\rangle_{g, \ell}=\int_{\overline{\mathcal{M}}_{g, \ell}} \psi_{1}^{n_{1}} \cdots \psi_{\ell}^{n_{\ell}} \lambda_{j} .
$$

Following $[8,15]$ we define a series of polynomials by a recursion formula

$$
\hat{\xi}_{n+1}(t)=t^{2}(t-1) \frac{d}{d t} \hat{\xi}_{n}(t)=D \hat{\xi}_{n}(t)
$$

with the initial condition $\hat{\xi}_{0}(t)=t-1$. The differential operator $D=t^{2}(t-$ 1) $\frac{d}{d t}$ found in [15, Example 4.1] simplifies many of the combinatorial difficulties of the linear Hodge integrals and Hurwitz numbers. The degree of $\hat{\xi}_{n}(t)$ is $(2 n+1)$. We consider symmetric polynomials of degree $3(2 g-2+\ell)$,

$$
\widehat{\mathcal{H}}_{g, \ell}\left(t_{1}, \ldots, t_{\ell}\right)=\sum_{n_{1}, \ldots, n_{\ell}}\left\langle\tau_{n_{1}} \cdots \tau_{n_{\ell}} \Lambda_{g}^{\vee}(1)\right\rangle_{g, \ell} \prod_{i=1}^{\ell} \hat{\xi}_{n_{i}}\left(t_{i}\right),
$$

where $\Lambda_{g}^{\vee}(1)=1-\lambda_{1}+\cdots+(-1)^{g} \lambda_{g}$. The following is our main theorem.

Theorem 1.1. The polynomial generating functions of the linear Hodge integrals (1.1) satisfy the following topological recursion formula:

$$
\begin{aligned}
(2 g & \left.-2+\ell+\sum_{i=1}^{\ell} \frac{1}{t_{i}} D_{i}\right) \widehat{\mathcal{H}}_{g, \ell}\left(t_{L}\right) \\
= & \sum_{i<j} \frac{t_{i}^{2} \hat{\xi}_{0}\left(t_{j}\right) D_{i} \widehat{\mathcal{H}}_{g, \ell-1}\left(t_{L \backslash\{j\}}\right)-t_{j}^{2} \hat{\xi}_{0}\left(t_{i}\right) D_{j} \widehat{\mathcal{H}}_{g, \ell-1}\left(t_{L \backslash\{i\}}\right)}{t_{i}-t_{j}} \\
& +\sum_{i=1}^{\ell}\left[D_{u_{1}} D_{u_{2}} \widehat{\mathcal{H}}_{g-1, \ell+1}\left(u_{1}, u_{2}, t_{L \backslash\{i\}}\right)\right]_{u_{1}=u_{2}=t_{i}} \\
& +\frac{1}{2} \sum_{i=1}^{\ell} \sum_{\substack{g_{1}+g_{2}=g \\
J \sqcup K=L \backslash\{i\}}}^{\text {stable }} D_{i} \widehat{\mathcal{H}}_{g_{1},|J|+1}\left(t_{i}, t_{J}\right) D_{i} \widehat{\mathcal{H}}_{g_{2},|K|+1}\left(t_{i}, t_{K}\right),
\end{aligned}
$$

where $D_{i}=t_{i}^{2}\left(t_{i}-1\right) \frac{\partial}{\partial t_{i}}$. The last summation is taken over all partitions $g=$ $g_{1}+g_{2}$ of the genus $g$ and disjoint union decompositions $J \sqcup K=L \backslash\{i\}$ satisfying the stability conditions $2 g_{1}-1+|J|>0$ and $2 g_{2}-1+|K|>0$. Here $L=\{1,2, \ldots, \ell\}$ is the index set, and for a subset $I \subset L$ we write $t_{I}=\left(t_{i}\right)_{i \in I}$. 
The recursion formula (1.2) is a topological recursion in the sense that it gives the generating function of linear Hodge integrals of complexity $2 g-2+\ell=n$ in terms of those of complexity $n-1$. The same topological structure appears in other recursion formulas such as those discussed in $[5,6,9,10,15,27-31]$.

We prove Theorem 1.1 by computing the Laplace transform of the Hurwitz number $h_{g, \mu}$ as a function of a partition $\mu$. Let $f: X \rightarrow \mathbb{P}^{1}$ be a morphism of connected nonsingular algebraic curve $X$ of genus $g$ onto the projective line defined over $\mathbb{C}$. If we regard $f$ as a meromorphic function on $X$, then the profile of $f$ is the list of orders of its poles being considered as a partition of the degree of $f$. The Hurwitz number $h_{g, \mu}$ we deal with in this paper is the number of topological types of $f$ of given genus $g$ and profile $\mu$ being counted with the weight $1 / \mid$ Aut $(f) \mid$. The celebrated cut-and-join equation of Goulden and Jackson [13] and Vakil [34] (which was essentially known to Hurwitz [19]) applied to the Laplace transformed Hurwitz numbers is exactly the polynomial recursion (1.2). The idea of taking the Laplace transform of the cut-and-join equation comes from [8]. It is shown in [8] that (1.2) implies the Bouchard-Mariño conjecture on the topological recursion for Hurwitz numbers [3], which is the simplest case of the more general conjecture on the closed and open Gromov-Witten invariants of toric Calabi-Yau 3-folds [2].

The significance of (1.2) being a polynomial is two-fold. Firstly, the leading coefficients of $\widehat{\mathcal{H}}_{g, \ell}$ are the $\psi$-class intersection numbers. It was proved by Okounkov and Pandharipande [32] that the large partition asymptotics of the Hurwitz numbers recover the Witten-Kontsevich theorem, i.e., the Virasoro constraint condition for the $\psi$-class intersection numbers $[6,23,35]$. Since the Laplace transform contains more information than the asymptotic behavior, the proof of the Witten conjecture [35] becomes just comparing the leading coefficients of the polynomial equation (1.2). The second significance is that the coefficients of the lowest degree terms are the linear Hodge integrals containing the $\lambda_{g}$-class. The topological recursion recovers the formula for $\left\langle\tau_{n_{1}} \cdots \tau_{n_{\ell}} \lambda_{g}\right\rangle_{g, \ell}$ in terms of $\left\langle\tau_{2 g-1} \lambda_{g}\right\rangle_{g, 1}$. We remark that the same polynomiality is observed in $[20,21]$ in the context of integrable systems.

We note that all the formulas in this paper have been more or less established in various different formulations [4, 14-16, 20,24]. Since (1.2) is equivalent to the cut-and-join equation, logically speaking one may say there is nothing new. The contribution of this paper is the simple expression of our formulation of the cut-and-join equation (3.14) and a new point of view of understanding (1.2) as the Laplace transform of (3.14). It gives a clear and unified picture of some of the results established in $[4,16,20]$. 
The paper is organized as follows. We begin with setting our notations and reviewing definitions of Hurwitz numbers in Section 2. In Section 3 we formulate the cut-and-join equation as a functional equation for functions in partitions. Although there are a large number of literature on the subject $[4,13-16,20,21,24,26,34,36]$, we provide a full detail in this section because we wish to arrive at a simpler formulation of the equation. We then introduce the idea of Laplace transformation following [8] in Section 4. Here the role of the Lambert curve, the spectral curve of the topological recursion for Hurwitz numbers introduced in $[1,3,8,9]$, is identified as the Riemann surface of a meromorphic function that is obtained by the Laplace transform. The following Section 5 establishes Theorem 1.1. In the final section we derive the Dijkgraaf-Verlinde-Verlinde (DVV) formula [6] for the WittenKontsevich theorem $[23,35]$ from $(1.2)$ as a simple corollary. We also give the combinatorial coefficient of the $\lambda_{g}$ formula $[11,12]$ from the topological recursion.

\section{Hurwitz numbers}

Let $X$ be a nonsingular complete algebraic curve of genus $g$ defined over the complex number field $\mathbb{C}$, and $f: X \rightarrow \mathbb{P}^{1}$ a morphism of $X$ to the projective line $\mathbb{P}^{1}$. If we regard $f$ a meromorphic function on the Riemann surface $X$, then the inverse image $f^{-1}(\infty)=\left\{p_{1}, \ldots, p_{\ell}\right\}$ of $\infty \in \mathbb{P}^{1}$ is the set of poles of $f$. We can name these $\ell$ points so that the list of pole orders becomes a partition $\mu=\left(\mu_{1} \geq \mu_{2} \geq \cdots \geq \mu_{\ell}>0\right)$ of the degree of the map. Thus the size of this partition $|\mu|=\mu_{1}+\cdots+\mu_{\ell}$ is $\operatorname{deg} f$, and its length $\ell(\mu)=\ell$ is the number of poles of $f$. Each part $\mu_{i}$ determines a local description of the map $f$, which is given by $z \longmapsto z^{\mu_{i}}$ in terms of a local coordinate $z$ of $X$ around $p_{i}$. A critical point, or a ramification point, of $f$ is a point $p \in X$ at which the derivative vanishes $d f(p)=0$, and $w=f(p)$ is a critical value, or a branched point of $f$. Let $B \subset \mathbb{P}^{1}$ be the set of all branched points of $f$. Then

$$
\left.f\right|_{f^{-1}\left(\mathbb{P}^{1} \backslash B\right)}: f^{-1}\left(\mathbb{P}^{1} \backslash B\right) \longrightarrow \mathbb{P}^{1} \backslash B
$$

is a topological covering of degree $|\mu|$. When the derivative $d f$ has a simple zero at $p$, we say $p$ is a simple ramification point of $f$. If over every branched point except for $\infty$ there is exactly one simple ramification point, then we call $f$ a Hurwitz cover. The partition $\mu$ gives the profile of a Hurwitz cover. The number $h_{g, \mu}$ of topological types of Hurwitz covers of given genus $g$ and profile $\mu$, counted with the weight factor $1 /|\operatorname{Aut} f|$, is the Hurwitz number we 
are interested in this paper. To be more precise, we study $h_{g, \mu}$ as a function of partition $\mu$. We will compute the Laplace transform of $h_{g, \mu}$ and find the equations that they satisfy.

Let $r$ denote the number of simple ramification points of $f$. This gives the dimension of the Hurwitz scheme, i.e., the moduli space of all Hurwitz covers for a given genus and a profile [32, Section 7.3.2]. Since (2.1) is a topological covering, the Euler characteristic of $f^{-1}\left(\mathbb{P}^{1} \backslash B\right)$ is given by

$$
\chi\left(f^{-1}\left(\mathbb{P}^{1} \backslash B\right)\right)=\operatorname{deg} f \cdot \chi\left(\mathbb{P}^{1} \backslash B\right)=|\mu|(1-r) .
$$

On the other hand, since $f^{-1}(x)$ contains exactly $\operatorname{deg} f-1$ points for every $x \in B \backslash\{\infty\}$ and since $f^{-1}(\infty)$ has $\ell$ points,

$$
\chi\left(f^{-1}\left(\mathbb{P}^{1} \backslash B\right)\right)=2-2 g(X)-\ell-r(|\mu|-1) .
$$

We thus obtain the Riemann-Hurwitz formula

$$
r=r(g, \mu)=2 g-2+\ell+|\mu|
$$

The celebrated Ekedahl-Lando-Shapiro-Vainshtein formula [7,17,32] relates Hurwitz numbers and linear Hodge integrals on the Deligne-Mumford moduli stack $\overline{\mathcal{M}}_{g, \ell}$ consisting of stable algebraic curves of genus $g$ with $\ell$ distinct nonsingular marked points subject to the stability condition $2 g-2+\ell>0$. Denote by $\pi_{g, \ell}: \overline{\mathcal{M}}_{g, \ell+1} \rightarrow \overline{\mathcal{M}}_{g, \ell}$ the natural projection and by $\omega_{\pi_{g, \ell}}$ the relative dualizing sheaf of the universal curve $\pi_{g, \ell}$. The Hodge bundle $\mathbb{E}$ on $\overline{\mathcal{M}}_{g, \ell}$ is defined by $\mathbb{E}=\left(\pi_{g, \ell}\right)_{*} \omega_{\pi_{g, \ell}}$, and the $\lambda$-classes are the Chern classes

$$
\lambda_{i}=c_{i}(\mathbb{E}) \in H^{2 i}\left(\overline{\mathcal{M}}_{g, \ell}, \mathbb{Q}\right)
$$

of the Hodge bundle. Let $\sigma_{i}: \overline{\mathcal{M}}_{g, \ell} \rightarrow \overline{\mathcal{M}}_{g, \ell+1}$ be the $i$ th tautological section of $\pi$, and put $\mathcal{L}_{i}=\sigma_{i}^{*}\left(\omega_{\pi_{g, \ell}}\right)$. The $\psi$-classes are defined by

$$
\psi_{i}=c_{1}\left(\mathcal{L}_{i}\right) \in H^{2}\left(\overline{\mathcal{M}}_{g, \ell}, \mathbb{Q}\right) .
$$

The linear Hodge integrals are rational numbers defined by

$$
\left\langle\tau_{n_{1}} \cdots \tau_{n_{\ell}} \lambda_{j}\right\rangle_{g, \ell}=\int_{\overline{\mathcal{M}}_{g, \ell}} \psi_{1}^{n_{1}} \cdots \psi_{\ell}^{n_{\ell}} \lambda_{j}
$$


which are 0 unless $n_{1}+\cdots+n_{\ell}+j=3 g-3+\ell$. Let us denote by $\Lambda_{g}^{\vee}(1)=$ $1-\lambda_{1}+\cdots+(-1)^{g} \lambda_{g}$. The ELSV formula states

$$
h_{g, \mu}=\frac{r(g, \mu) !}{|\operatorname{Aut}(\mu)|} \prod_{i=1}^{\ell(\mu)} \frac{\mu_{i}^{\mu_{i}}}{\mu_{i} !} \int_{\overline{\mathcal{M}}_{g, \ell(\mu)}} \frac{\Lambda_{g}^{\vee}(1)}{\prod_{i=1}^{\ell(\mu)}\left(1-\mu_{i} \psi_{i}\right)},
$$

where $\operatorname{Aut}(\mu)$ is the permutation group that interchanges the equal parts of $\mu$. The appearance of this automorphism factor is due to the difference between giving a profile $\mu$ and naming all points in $f^{-1}(\infty)$. If all parts of $\mu$ are distinct, then the poles of $f$ are naturally labeled by the pole order. But when two or more parts are the same, there is no way to distinguish the Hurwitz covers obtained by interchanging these poles of the same order. The factor $1 /|\operatorname{Aut}(\mu)|$ takes care of this overount.

Although $\overline{\mathcal{M}}_{g, \ell}$ is defined as the moduli stack of stable curves satisfying the stability condition $2-2 g-\ell<0$, Hurwitz numbers are well defined for unstable geometries $(g, \ell)=(0,1)$ and $(0,2)$. It is an elementary exercise to show that

$$
h_{0, k}=k^{k-3} \quad \text { and } \quad h_{0,\left(\mu_{1}, \mu_{2}\right)}=\frac{\left(\mu_{1}+\mu_{2}\right) !}{\mu_{1}+\mu_{2}} \quad \frac{\mu_{1}^{\mu_{1}}}{\mu_{1} !} \frac{\mu_{2}^{\mu_{2}}}{\mu_{2} !} .
$$

The ELSV formula remains true for unstable cases by defining

$$
\begin{gathered}
\int_{\overline{\mathcal{M}}_{0,1}} \frac{\Lambda_{0}^{\vee}(1)}{1-k \psi}=\frac{1}{k^{2}}, \\
\int_{\overline{\mathcal{M}}_{0,2}} \frac{\Lambda_{0}^{\vee}(1)}{\left(1-\mu_{1} \psi_{1}\right)\left(1-\mu_{2} \psi_{2}\right)}=\frac{1}{\mu_{1}+\mu_{2}} .
\end{gathered}
$$

\section{The cut-and-join equation}

The Hurwitz numbers satisfy a set of combinatorial equations called the cutand-join equation of $[13,34]$. It is essentially the same relation Hurwitz dealt with in his seminal paper [19]. Due to the modern formulation in these more recent papers, the combinatorial equation has become an effective tool of algebraic geometry for studying Hurwitz numbers and many related subjects $[4,14-18,21,26,32,36]$. In this section we review the equation following $[13,24,34,36]$ and give its simplest formulation that is suitable to compute its Laplace transform in Section 5. 
The topological covering (2.1) gives rise to a unique point in the character variety

$$
\rho \in \operatorname{Hom}\left(\pi_{1}\left(\mathbb{P}^{1} \backslash B\right), S_{d}\right) / S_{d},
$$

where $S_{d}$ is the symmetric group of $d=|\mu|$ letters and its action on the set of homomorphisms is through conjugation. Since the character variety classifies all topological coverings, we need to determine the condition for a covering to be a Hurwitz cover. Let us list the $r+1$ points in $B$ as

$$
B=\left\{x_{1}, \ldots, x_{r}, \infty\right\}
$$

Choose a base point $*$ on $\mathbb{P}^{1} \backslash B$, and denote by $\gamma_{k}$ a closed path starting from $*$ that goes around $x_{k}$ in the positive direction, and comes back to $*$. The loop $\gamma_{\infty}$ is the loop going around $\infty$. Then up to conjugation, we have

$$
\pi_{1}\left(\mathbb{P}^{1} \backslash B\right) \cong\left\langle\gamma_{1}, \ldots, \gamma_{r}, \gamma_{\infty} \mid \gamma_{1} \cdots \gamma_{r} \cdot \gamma_{\infty}=1\right\rangle
$$

Now recall that over each $x_{k}$ there is only one ramification point, say $p_{k}$, which is simple. Therefore, in terms of the representation $\rho$ corresponding to the Hurwitz cover $f$, the generator $\gamma_{k}$ is mapped to a transposition $(a b) \in S_{d}$. Next, recall that the ramification behavior over $\infty$ is determined by the profile $\mu$, and that each part $\mu_{i}$ determines the map $f$ locally as $z \longmapsto z^{\mu_{i}}$. In terms of the representation, this means that

$$
\rho\left(\gamma_{\infty}\right)=c_{1} c_{2} \cdots c_{\ell}
$$

where

$$
c_{1} \sqcup \cdots \sqcup c_{\ell}=\{1,2, \ldots, d\}
$$

is a disjoint cycle decomposition of the index set and each $c_{i}$ is a cycle of length $\mu_{i}$.

The cut-and-join equation represents the number of Hurwitz covers of a given genus $g$ and profile $\mu$ in terms of those with profiles obtained by either cutting a part into two pieces, or joining two parts together. Let $p \in X$ be a point at which the covering $f: X \rightarrow \mathbb{P}^{1}$ is simply ramified. Locally we can name sheets, so we assume sheets $a$ and $b$ are ramified over $x_{r}=f(p) \in$ $B \subset \mathbb{P}^{1}$. In terms of the representation we have $\rho\left(\gamma_{r}\right)=(a b) \in S_{d}$. When we merge $x_{r}$ to $\infty$, the generators $\gamma_{r}$ and $\gamma_{\infty}$ of $\pi_{1}\left(\mathbb{P}^{1} \backslash B\right)$ are replaced by their product $\gamma_{r} \gamma_{\infty}$. The representation $\rho$ maps this generator to $(a b) c_{1} \cdots c_{\ell}$. Now one of the two things happen: 
1. The cut case, in which both sheets are ramified at the same point $p_{i}$ of the inverse image $f^{-1}(\infty)=\left\{p_{1}, \ldots, p_{\ell}\right\}$. In terms of $\rho$, this means both indices $a$ and $b$ are contained in the same cycle $c_{i}$. Since $c_{1}, \ldots, c_{\ell}$ are disjoint, we only need to calculate $(a b) c_{i}$. By re-naming all the sheets and assuming $a<b=a+\alpha<\mu_{i}=\alpha+\beta$, we can compute

$$
\begin{aligned}
& (a[a+\alpha])(12 \cdots[a-1] a[a+1] \cdots[a+\alpha] \cdots[\alpha+\beta]) \\
& =(a[a+1] \cdots[a+\alpha-1])([a+\alpha][a+\alpha+1] \\
& \quad \cdots[\alpha+\beta] 12 \cdots[a-1]) .
\end{aligned}
$$

The result is the product of two disjoint cycles of length $\alpha$ and $\beta$. Thus, the merging eliminates a profile $\mu$ and creates a new profile

$$
\left(\mu_{1}, \ldots, \widehat{\mu_{i}}, \ldots, \mu_{\ell}, \alpha, \beta\right)=(\mu(\hat{i}), \alpha, \beta)
$$

of length $\ell+1$. Here the ${ }^{\widehat{ }} \operatorname{sign}$ means removing the entry. Note that the size of the partition $|\mu|$ is unchanged, because it is the degree of the map $f$. When $\alpha$ is chosen, the total number of such cuttings is $\alpha+\beta$ because this is the number of choices for $a$ in the index set $\{1,2, \ldots, \alpha+\beta\}$. We also note that when $\alpha=\beta$, the number is actually $\alpha$, instead of $\alpha+\beta$.

2. The join case, in which sheets $a$ and $b$ are ramified at two distinct points, say $p_{i}$ and $p_{j}$, above $\infty$. In other words, $a \in c_{i}$ and $b \in c_{j}$. Again by re-numbering, we can calculate

$$
\begin{aligned}
(a b) & \left(12 \cdots[a-1] a[a+1] \cdots \mu_{i}\right)\left(\left[\mu_{i}+1\right]\right. \\
& \left.\cdots[b-1] b[b+1] \cdots\left[\mu_{i}+\mu_{j}\right]\right) \\
= & \left(12 \cdots[a-1] b[b+1] \cdots\left[\mu_{i}+\mu_{j}\right]\left[\mu_{i}+1\right]\right. \\
& \left.\cdots[b-1] a[a+1] \cdots \mu_{i}\right) .
\end{aligned}
$$

Thus the result of merging creates a new profile

$$
\left(\mu_{1}, \ldots, \widehat{\mu_{i}}, \ldots, \widehat{\mu_{j}}, \ldots, \mu_{\ell}, \mu_{i}+\mu_{j}\right)=\left(\mu(\hat{i}, \hat{j}), \mu_{i}+\mu_{j}\right)
$$

of length $\ell-1$ and size $|\mu|$. The total number of ways to make the join is $\mu_{i} \mu_{j}$, because we have $\mu_{i}$-choices for $a$ and $\mu_{j}$-choices for $b$.

To utilize the above consideration into Hurwitz numbers, let us introduce the generating function of Hurwitz numbers

$$
\mathbf{H}(s, \mathbf{p})=\sum_{g \geq 0} \sum_{\ell \geq 1} \mathbf{H}_{g, \ell}(s, \mathbf{p}) ; \quad \mathbf{H}_{g, \ell}(s, \mathbf{p})=\sum_{\mu: \ell(\mu)=\ell} h_{g, \mu} \mathbf{p}_{\mu} \frac{s^{r(g, \mu)}}{r(g, \mu) !},
$$


where $\mathbf{p}_{\mu}=p_{\mu_{1}} p_{\mu_{2}} \cdots p_{\mu_{\ell}}$, and $r(g, \mu)$ is the number of simple ramification points (2.2). The summation in $\mathbf{H}_{g, \ell}(s, \mathbf{p})$ is over all partitions of length $\ell$. Here $p_{1}, p_{2}, p_{3}, \ldots$ are parameters that encode the information of partitions. The other parameter $s$ counts the number $r$ of simple ramification points. Since $r$ and $\mu$ recover the genus $g, s$ is a topological parameter. Note that merging $x_{r}$ to $\infty$ means decreasing $r$ by 1 , or differentiating the generating function with respect to $s$. The result of this differentiation is the cut and join operations discussed above. Here we need to note that the cut cases may cause a disconnected covering of $\mathbb{P}^{1}$. Recall that the exponential generating function

$$
\mathrm{e}^{\mathbf{H}(s, \mathbf{p})}=1+\mathbf{H}(s, \mathbf{p})+\frac{1}{2} \mathbf{H}(s, \mathbf{p})^{2}+\frac{1}{3 !} \mathbf{H}(s, \mathbf{p})^{3}+\cdots
$$

counts disconnected Hurwitz coverings. The power of $\mathbf{H}(s, \mathbf{p})$ is the number of connected components. Now the above merging consideration gives the following equation, which is the cut-and-join equation as a linear partial differential equation

$$
\left[\frac{\partial}{\partial s}-\frac{1}{2} \sum_{\alpha, \beta \geq 1}\left((\alpha+\beta) p_{\alpha} p_{\beta} \frac{\partial}{\partial p_{\alpha+\beta}}+\alpha \beta p_{\alpha+\beta} \frac{\partial^{2}}{\partial p_{\alpha} \partial p_{\beta}}\right)\right] \mathrm{e}^{\mathbf{H}(s, \mathbf{p})}=0 .
$$

We can immediately deduce

$$
\frac{\partial \mathbf{H}}{\partial s}=\frac{1}{2} \sum_{\alpha, \beta \geq 1}\left((\alpha+\beta) p_{\alpha} p_{\beta} \frac{\partial \mathbf{H}}{\partial p_{\alpha+\beta}}+\alpha \beta p_{\alpha+\beta} \frac{\partial^{2} \mathbf{H}}{\partial p_{\alpha} \partial p_{\beta}}+\alpha \beta p_{\alpha+\beta} \frac{\partial \mathbf{H}}{\partial p_{\alpha}} \cdot \frac{\partial \mathbf{H}}{\partial p_{\beta}}\right)
$$

This is the cut-and-join equation for the generating function $\mathbf{H}(s, \mathbf{p})$ of the number of connected Hurwitz coverings.

At this stage, we apply the ELSV formula (2.3) to (3.2). For a partition $\mu$ of length $\ell$, we define

$$
\begin{aligned}
H_{g}(\mu) & =\frac{|\operatorname{Aut}(\mu)|}{r(g, \mu) !} \cdot h_{g, \mu} \\
& =\sum_{n_{1}+\cdots+n_{\ell} \leq 3 g-3+\ell}\left\langle\tau_{n_{1}} \cdots \tau_{n_{\ell}} \Lambda_{g}^{\vee}(1)\right\rangle \prod_{i=1}^{\ell} \frac{\mu_{i}^{\mu_{i}+n_{i}}}{\mu_{i} !}
\end{aligned}
$$


Then we have

$$
\mathbf{H}_{g, \ell}(s, \mathbf{p})=\sum_{\mu: \ell(\mu)=\ell} \frac{1}{|\operatorname{Aut}(\mu)|} H_{g}(\mu) \mathbf{p}_{\mu} s^{r(g, \mu)}=\frac{1}{\ell !} \sum_{\left(\mu_{1}, \ldots, \mu_{\ell}\right) \in \mathbb{N} \ell} H_{g}(\mu) \mathbf{p}_{\mu} s^{r(g, \mu)} .
$$

The automorphism factor $|\operatorname{Aut}(\mu)|$ in the formula comes from the resummation. For any function $f(\mu)$ in $\mu$, we have a change of summation formula

$$
\sum_{\mu \in \mathbb{N}^{\ell}} f(\mu)=\sum_{\mu: \ell(\mu)=\ell} \frac{1}{|\operatorname{Aut}(\mu)|} \sum_{\sigma \in S_{\ell}} f\left(\mu^{\sigma}\right)
$$

where $S_{\ell}$ is the permutation group of $\ell$ letters and

$$
\mu^{\sigma}=\left(\mu_{\sigma(1)}, \ldots, \mu_{\sigma(\ell)}\right) \in \mathbb{N}^{\ell}
$$

is the integer vector obtained by permuting the parts of $\mu$ by $\sigma \in S_{\ell}$. If $f(\mu)$ is a symmetric function, then the summation over $S_{\ell}$ simply contributes $\ell$ ! to the formula, as in (3.6). For a partition $\mu$, let us denote by $m_{\alpha}(\mu)$ the multiplicity of $\alpha$ in $\mu$, i.e., the number of $\alpha$ repeated in $\mu$. Then we have

$$
|\operatorname{Aut}(\mu)|=\prod_{k \geq 1} m_{k}(\mu) !
$$

Let us now compare the coefficient of $\mathbf{p}_{\mu} s^{r-1}$ in the cut-and-join equation (3.4) for a given partition $\mu$ and an integer $r \geq 1$. The left-hand side contributes

$$
r(g, \mu) \frac{H_{g}(\mu)}{|\operatorname{Aut}(\mu)|},
$$

subject to the condition $r=r(g, \mu)$.

The terms of $\mathbf{p}_{\mu} s^{r-1}$ that come from the cut-operation of the right-hand side of (3.4) must have a profile $\left(\mu(\hat{i}, \hat{j}), \mu_{i}+\mu_{j}\right)$, because

$$
\begin{aligned}
r\left(g,\left(\mu(\hat{i}, \hat{j}), \mu_{i}+\mu_{j}\right)\right) & =2 g-2+\ell\left(\mu(\hat{i}, \hat{j}), \mu_{i}+\mu_{j}\right)+\left|\left(\mu(\hat{i}, \hat{j}), \mu_{i}+\mu_{j}\right)\right| \\
& =2 g-2+(\ell-1)+|\mu|=r(g, \mu)-1 .
\end{aligned}
$$

We see that the application of the differential operator $p_{\mu_{i}} p_{\mu_{j}} \partial / \partial p_{\mu_{i}+\mu_{j}}$ to $\mathbf{H}(s, \mathbf{p})$ restores the profile $\mu$ from $\left(\mu(\hat{i}, \hat{j}), \mu_{i}+\mu_{j}\right)$. Thus the coefficient of 
$\mathbf{p}_{\mu} s^{r-1}$ is

$$
\frac{1}{|\operatorname{Aut}(\mu)|} \sum_{i<j}\left(\mu_{i}+\mu_{j}\right) H_{g}\left(\mu(\hat{i}, \hat{j}), \mu_{i}+\mu_{j}\right)
$$

In this consideration, we are naming all parts of $\mu$ to apply the cut-operation. Therefore, we need to compensate the overcount by the $\operatorname{Aut}(\mu)$-factor. In terms of combinatorics, we can obtain (3.10) in a different way. It is easy to see [36, Section 2.3] that

$$
\left|\operatorname{Aut}\left(\mu(\hat{i}, \hat{j}), \mu_{i}+\mu_{j}\right)\right|=\left\{\begin{array}{l}
|\operatorname{Aut}(\mu)| \frac{m_{\mu_{i}+\mu_{j}}(\mu)+1}{m_{\mu_{i}}(\mu) m_{\mu_{j}}(\mu)}, \quad \mu_{i} \neq \mu_{j}, \\
|\operatorname{Aut}(\mu)| \frac{m_{\mu_{i}+\mu_{j}}(\mu)+1}{m_{\mu_{i}}(\mu)\left(m_{\mu_{i}}(\mu)-1\right)}, \quad \mu_{i}=\mu_{j} .
\end{array}\right.
$$

So if $\mu_{i} \neq \mu_{j}$, then

$$
\begin{aligned}
& \frac{1}{|\operatorname{Aut}(\mu)|}\left(\mu_{i}+\mu_{j}\right) H_{g}\left(\mu(\hat{i}, \hat{j}), \mu_{i}+\mu_{j}\right) \\
& =\left(\mu_{i}+\mu_{j}\right) \frac{m_{\mu_{i}+\mu_{j}}(\mu)+1}{m_{\mu_{i}}(\mu) m_{\mu_{j}}(\mu)} \frac{H_{g}\left(\mu(\hat{i}, \hat{j}), \mu_{i}+\mu_{j}\right)}{\left|\operatorname{Aut}\left(\mu(\hat{i}, \hat{j}), \mu_{i}+\mu_{j}\right)\right|},
\end{aligned}
$$

where each factor of the right-hand side has combinatorial significance. When $\mu_{i}=\mu_{j}=\alpha$, we have

$$
\frac{1}{|\operatorname{Aut}(\mu)|}\left(\mu_{i}+\mu_{j}\right) H_{g}\left(\mu(\hat{i}, \hat{j}), \mu_{i}+\mu_{j}\right)=\alpha \frac{m_{2 \alpha}(\mu)+1}{\left(\begin{array}{c}
m_{\alpha}(\mu) \\
2
\end{array}\right)} \frac{H_{g}(\mu(\hat{i}, \hat{j}), 2 \alpha)}{|\operatorname{Aut}(\mu(\hat{i}, \hat{j}), 2 \alpha)|}
$$

where the part $\alpha$ is removed from the $i$ th and $j$ th slots of $\mu$.

In a join term we must have a profile $(\mu(\hat{i}), \alpha, \beta)$. Since $\ell(\mu(\hat{i}), \alpha, \beta)=$ $\ell+1$, changing $r$ to $r-1$ requires reducing the genus. One possibility is

$$
\begin{aligned}
r(g-1,(\mu(\hat{i}), \alpha, \beta)) & =2(g-1)-2+\ell(\mu(\hat{i}), \alpha, \beta)+|(\mu(\hat{i}), \alpha, \beta)| \\
& =2 g-2+(\ell+1)+|\mu|-2=r(g, \mu)-1 .
\end{aligned}
$$

In this case the differential operator $p_{\alpha+\beta} \partial^{2} / \partial p_{\alpha} \partial p_{\beta}$ applied to $\mathbf{H}(s, \mathbf{p})$ recovers the profile $\mu$. The coefficient of $\mathbf{p}_{\mu} s^{r-1}$ is then

$$
\frac{1}{2|\operatorname{Aut}(\mu)|} \sum_{i=1}^{\ell} \sum_{\alpha+\beta=\mu_{i}} \alpha \beta H_{g-1}(\mu(\hat{i}), \alpha, \beta)
$$


Here again we can give a combinatorial explanation of this formula using (3.8) and (3.11). When $\alpha \neq \beta$, we have

$$
\begin{aligned}
\frac{1}{|\operatorname{Aut}(\mu)|} \alpha \beta H_{g-1}(\mu(\hat{i}), \alpha, \beta)= & \alpha \beta \frac{\left(m_{\alpha}(\mu)+1\right)\left(m_{\beta}(\mu)+1\right)}{m_{\mu_{i}}(\mu)} \\
& \times \frac{H_{g-1}(\mu(\hat{i}), \alpha, \beta)}{|\operatorname{Aut}(\mu(\hat{i}), \alpha, \beta)|} .
\end{aligned}
$$

And if $\alpha=\beta=\frac{1}{2} \mu_{i}$, then

$$
\frac{1}{|\operatorname{Aut}(\mu)|} \alpha^{2} H_{g-1}(\mu(\hat{i}), \alpha, \alpha)=2 \alpha^{2} \frac{\left(\begin{array}{l}
m_{\alpha}(\mu)+2 \\
2
\end{array}\right)}{m_{\mu_{i}}(\mu)} \frac{H_{g-1}(\mu(\hat{i}), \alpha, \alpha)}{|\operatorname{Aut}(\mu(\hat{i}), \alpha, \alpha)|} .
$$

The overall factor 2 in the right-hand side comes from the second-order differentiation $\partial^{2} / \partial p_{\alpha}^{2}$.

There is yet another possibility to obtain the profile $\mu$ from a joinoperation, if we utilize disconnected Hurwitz covers. Consider Hurwitz covers

$$
f_{1}: X_{1} \longrightarrow \mathbb{P}^{1} \quad \text { and } \quad f_{2}: X_{2} \longrightarrow \mathbb{P}^{1}
$$

of genus $g_{1}$ (resp. $\left.g_{2}\right)$ and profile $\left(\nu_{1}, \alpha\right)\left(\right.$ resp. $\left.\left(\nu_{2}, \beta\right)\right)$. Let $\nu_{1} \sqcup \nu_{2}$ denote the partition obtained by gathering all parts of $\nu_{1}$ and $\nu_{2}$ together. If $g_{1}+g_{2}=g$ and $\nu_{1} \sqcup \nu_{2}=\mu(\hat{i})$, then the join-operation recovers the profile $\mu$, provided that $\alpha+\beta=\mu_{i}$. This is because

$$
\begin{aligned}
r\left(g_{1},\left(\nu_{1}, \alpha\right)\right) & =2 g_{1}-2+\ell\left(\nu_{1}\right)+1+\left|\nu_{1}\right|+\alpha, \\
r\left(g_{2},\left(\nu_{2}, \beta\right)\right) & =2 g_{2}-2+\ell\left(\nu_{2}\right)+1+\left|\nu_{2}\right|+\beta, \\
r(g, \mu)-1 & =2 g-2+\ell+|\mu|-1 .
\end{aligned}
$$

The $\mathbf{p}_{\mu} s^{r-1}$-term comes from $p_{\alpha+\beta} \frac{\partial \mathbf{H}}{\partial p_{\alpha}} \cdot \frac{\partial \mathbf{H}}{\partial p_{\beta}}$, and its coefficient is

$$
\frac{1}{2|\operatorname{Aut}(\mu)|} \sum_{i=1}^{\ell} \sum_{\alpha+\beta=\mu_{i}} \alpha \beta \sum_{\substack{g_{1}+g_{2}=g \\ \nu_{1} \sqcup \nu_{2}=\mu(\hat{i})}} H_{g_{1}}\left(\nu_{1}, \alpha\right) H_{g_{2}}\left(\nu_{2}, \beta\right) .
$$

The combinatorial derivation of this formula follows from the identity

$$
\left|\operatorname{Aut}\left(\nu_{1} \sqcup \nu_{2}\right)\right|=\left|\operatorname{Aut}\left(\nu_{1}\right)\right|\left|\operatorname{Aut}\left(\nu_{2}\right)\right| \cdot \prod_{k \geq 1}\left(\begin{array}{c}
m_{k}\left(\nu_{1} \sqcup \nu_{2}\right) \\
m_{k}\left(\nu_{1}\right)
\end{array}\right) .
$$


When $\alpha \neq \beta$, we have

$$
\begin{aligned}
& \frac{1}{|\operatorname{Aut}(\mu)|} \alpha \beta \sum_{\substack{g_{1}+g_{2}=g \\
\nu_{1} \sqcup \nu_{2}=\mu(\hat{i})}} H_{g_{1}}\left(\nu_{1}, \alpha\right) H_{g_{2}}\left(\nu_{2}, \beta\right) \\
& =\alpha \beta \frac{\left(m_{\alpha}(\mu)+1\right)\left(m_{\beta}(\mu)+1\right)}{m_{\mu_{i}}(\mu)} \frac{1}{\prod_{k \geq 1}\left(\begin{array}{c}
m_{k}(\mu(\hat{i}), \alpha, \beta) \\
m_{k}\left(\nu_{1}, \alpha\right)
\end{array}\right)} \\
& \quad \times \frac{H_{g_{1}}\left(\nu_{1}, \alpha\right)}{\left|\operatorname{Aut}\left(\nu_{1}, \alpha\right)\right|} \frac{H_{g_{2}}\left(\nu_{2}, \beta\right)}{\left|\operatorname{Aut}\left(\nu_{2}, \beta\right)\right|} .
\end{aligned}
$$

And if $\alpha=\beta=\frac{1}{2} \mu_{i}$, then

$$
\begin{aligned}
& \frac{1}{|\operatorname{Aut}(\mu)|} \alpha^{2} \sum_{\substack{g_{1}+g_{2}=g \\
\nu_{1} \sqcup \nu_{2}=\mu(\hat{i})}} H_{g_{1}}\left(\nu_{1}, \alpha\right) H_{g_{2}}\left(\nu_{2}, \alpha\right) \\
& =2 \alpha^{2} \frac{\left(\begin{array}{c}
m_{\alpha}(\mu)+2 \\
2
\end{array}\right)}{m_{\mu_{i}}(\mu)} \frac{1}{\prod_{k \geq 1}\left(\begin{array}{c}
m_{k}(\mu(\hat{i}), \alpha, \alpha) \\
m_{k}\left(\nu_{1}, \alpha\right)
\end{array}\right)} \frac{H_{g_{1}}\left(\nu_{1}, \alpha\right)}{\left|\operatorname{Aut}\left(\nu_{1}, \alpha\right)\right|} \frac{H_{g_{2}}\left(\nu_{2}, \alpha\right)}{\left|\operatorname{Aut}\left(\nu_{2}, \alpha\right)\right|} .
\end{aligned}
$$

Assembling (3.10), (3.12) and (3.13) together, we obtain the combinatorial form of the cut-and-join equation.

Theorem 3.1 (Cut-and-join equation). The functions $H_{g}(\mu)$ of (3.5) satisfy a recursion equation

$$
\begin{aligned}
r(g, \mu) H_{g}(\mu)= & \sum_{i<j}\left(\mu_{i}+\mu_{j}\right) H_{g}\left(\mu(\hat{i}, \hat{j}), \mu_{i}+\mu_{j}\right)+\frac{1}{2} \sum_{i=1}^{\ell} \sum_{\alpha+\beta=\mu_{i}} \alpha \beta \\
& \times\left(H_{g-1}(\mu(\hat{i}), \alpha, \beta) \sum_{\substack{g_{1}+g_{2}=g \\
\nu_{1} \sqcup \nu_{2}=\mu(\hat{i})}} H_{g_{1}}\left(\nu_{1}, \alpha\right) H_{g_{2}}\left(\nu_{2}, \beta\right)\right) .
\end{aligned}
$$

\section{Laplace transform and the Lambert curve}

Since linear Hodge integrals $\left\langle\tau_{n_{1}} \cdots \tau_{n_{\ell}} \lambda_{j}\right\rangle$ do not depend on a partition $\mu$, it is natural to ask if there is any direct recursion formula for them without any reference to partitions. The answer is yes, and we give the formula in Section 5. The natural complexity measure for the moduli space $\overline{\mathcal{M}}_{g, \ell}$ is the absolute value $2 g-2+\ell$ of the Euler characteristic of an $\ell$-punctured 
Riemann surface of genus $g$. An inductive formula associated to $\overline{\mathcal{M}}_{g, \ell}$ with respect to $2 g-2+\ell$ is generally called a topological recursion. We wish to establish a topological recursion for linear Hodge integrals. In the light of (3.5) and the combinatorial cut-and-join equation (3.14), it is obvious what we should do to eliminate the $\mu$-dependence: just take the summation over all partitions $\mu$. This is the idea of the Laplace transform discovered in [8]. In this section we explain this idea.

Since the sum of $\frac{k^{k+n}}{k !}$ for all positive integer $k$ diverges, we are naturally led to the idea of Laplace transformation. Indeed,

$$
f_{n}(w)=\sum_{k=1}^{\infty} \frac{k^{k+n}}{k !} \mathrm{e}^{-k(w+1)}
$$

is a holomorphic function in $w$ for $\operatorname{Re}(w)>0$. This follows from Stirling's formula

$$
\mathrm{e}^{-k} \frac{k^{k+n}}{k !} \sim \frac{1}{\sqrt{2 \pi}} k^{n-\frac{1}{2}} \quad \text { for } \quad k \gg 1 .
$$

Note that the continuous estimate for (4.1) is given by

$$
\int_{0}^{\infty} x^{n-\frac{1}{2}} \mathrm{e}^{-x w} d x=\frac{\Gamma\left(n+\frac{1}{2}\right)}{w^{n+\frac{1}{2}}}
$$

for $n>-\frac{1}{2}$. Thus $f_{n}(w)$ of $(4.1)$ is expected to be a function of $\sqrt{w}$, instead of $w$ itself, if $n$ is an integer. We now come to the point of asking: what is the Riemann surface of the function $f_{n}(w)$ ? If the estimate (4.2) were exact, then the Riemann surface of $f_{n}(w)$ would have been the same as that of $\sqrt{w}$. But since it is not, we need a different idea.

The idea used in $[8]$ is the following. First, we introduce a function

$$
t=t(w)=1+\sum_{k=1}^{\infty} \frac{k^{k}}{k !} \mathrm{e}^{-k(w+1)},
$$

which is holomorphic for $\operatorname{Re}(w)>0$, and define

$$
x=\mathrm{e}^{-(w+1)} \quad \text { and } \quad y=\frac{t-1}{t} .
$$

We can solve $t=t(w)$ in terms of $x$ and $y$. The result is

$$
x=y \mathrm{e}^{-y} .
$$


Let us call the plane analytic curve

$$
C=\left\{(x, y) \in \mathbb{C}^{2} \mid x=y \mathrm{e}^{-y}\right\} \subset \mathbb{C}^{2}
$$

the Lambert curve. This naming is due to the resemblance of (4.3) and the classical Lambert $W$-function

$$
W(x)=-\sum_{k=1}^{\infty} \frac{k^{k-1}}{k !}(-x)^{k}
$$

The Lambert curve $C$ is analytically isomorphic to $\mathbb{C}$, so it is an open Riemann surface of genus 0 . The $x$-projection $\pi: C \rightarrow \mathbb{C}$ has a unique critical point $q_{0}=\left(\mathrm{e}^{-1}, 1\right) \in C$. In terms of the coordinates $w$ and $t$, the inverse function of (4.3), or the equation for the Lambert curve, is given by

$$
w=w(t)=-\frac{1}{t}-\log \left(1-\frac{1}{t}\right)=\sum_{m=2}^{\infty} \frac{1}{m} \frac{1}{t^{m}}
$$

which is holomorphic for $\operatorname{Re}(t)>1$. The critical point of the projection $\pi$ in this coordinate is $(w, t)=(0, \infty)$. Since the infinite series of $(4.7)$ starts at $m=2, \pi$ is locally a double-sheeted covering around $w=0$. And this is what we wanted. Indeed, the Lambert curve $C$ is the Riemann surface of the function $f_{n}(w)$. It is natural to consider $f_{n}(w)$ as a function in $t$, since $t$ is a global coordinate of $C$. So we re-define

$$
\hat{\xi}_{n}(t)=\sum_{k=1}^{\infty} \frac{k^{k+n}}{k !} \mathrm{e}^{-k(w+1)}
$$

which is simply $f_{n}(w)$ in terms of $t$ satisfying $w=w(t)$. But something remarkable happens here: $\hat{\xi}_{n}(t)$ is a polynomial in $t$ if $n \geq 0$. The proof is obvious. A standard property of the Laplace transform gives

$$
-\frac{d}{d w} f_{n}(w)=\sum_{k=1}^{\infty} \frac{k^{k+n+1}}{k !} \mathrm{e}^{-k(w+1)}=f_{n+1}(w),
$$

and the coordinate change (4.7) implies

$$
-\frac{d}{d w}=t^{2}(t-1) \frac{d}{d t}
$$


Therefore, $\hat{\xi}_{n}(t)$ 's satisfy a recursion formula

$$
\hat{\xi}_{n+1}(t)=t^{2}(t-1) \frac{d}{d t} \hat{\xi}_{n}(t)=D \hat{\xi}_{n}(t)
$$

Since $\hat{\xi}_{0}(t)=t-1$ from $(4.3)$, we see that $\hat{\xi}_{n}(t)$ is a polynomial in $t$ of degree $2 n+1$. It immediately follows that the Laplace transform

$$
\begin{aligned}
\widehat{\mathcal{H}}_{g, \ell}\left(t_{1}, \ldots, t_{\ell}\right) & =\sum_{\mu \in \mathbb{N}^{\ell}} H_{g}(\mu) \mathrm{e}^{-\left(\mu_{1}\left(w_{1}+1\right)+\cdots+\mu_{\ell}\left(w_{\ell}+1\right)\right)} \\
& =\sum_{n_{1}+\cdots+n_{\ell} \leq 3 g-3+\ell}\left\langle\tau_{n_{1}} \cdots \tau_{n_{\ell}} \Lambda_{g}^{\vee}(1)\right\rangle \prod_{i=1}^{\ell} \hat{\xi}_{n_{i}}\left(t_{i}\right)
\end{aligned}
$$

of $H_{g}(\mu)$ of $(3.5)$ is a symmetric polynomial in the $t$-variables and naturally lives on $C^{\ell}$, when $2 g-2+\ell>0$.

The unstable geometries $(g, \ell)=(0,1)$ and $(0,2)$ are the exceptions for this general formula. Recall the $(0,1)$ case $(2.4)$. We have

$$
\widehat{\mathcal{H}}_{0,1}(t)=\sum_{k=1}^{\infty} \frac{k^{k-2}}{k !} \mathrm{e}^{-k(w+1)}=-\frac{1}{2 t^{2}}+c=\hat{\xi}_{-2}(t)
$$

where the constant $c$ is given by

$$
c=\sum_{k=1}^{\infty} \frac{k^{k-2}}{k !} \mathrm{e}^{-k}
$$

The $(0,2)$ case $(2.5)$ is quite more involved. It is proved [8, Proposition 3.6] that we have

$$
\begin{aligned}
\widehat{\mathcal{H}}_{0,2}\left(t_{1}, t_{2}\right) & =\sum_{\mu_{1}, \mu_{2} \geq 1} \frac{1}{\mu_{1}+\mu_{2}} \cdot \frac{\mu_{1}^{\mu_{1}}}{\mu_{1} !} \cdot \frac{\mu_{2}^{\mu_{2}}}{\mu_{2} !} \mathrm{e}^{-\mu_{1}\left(w_{1}+1\right)} \mathrm{e}^{-\mu_{2}\left(w_{2}+1\right)} \\
& =\log \left(\frac{\hat{\xi}_{-1}\left(t_{1}\right)-\hat{\xi}_{-1}\left(t_{2}\right)}{x_{1}-x_{2}}\right)-\hat{\xi}_{-1}\left(t_{1}\right)-\hat{\xi}_{-1}\left(t_{2}\right),
\end{aligned}
$$

where

$$
\hat{\xi}_{-1}(t)=\frac{t-1}{t}=y
$$




\section{The topological recursion as a Laplace transform}

In the previous section we have computed the Laplace transform of $H_{g}(\mu)$ as a function on partitions $\mu$. In this section, we calculate the Laplace transform of the cut-and-join Equation (3.14) and prove Theorem 1.1.

Let us denote

$$
\langle\mu, w+1\rangle=\mu_{1}\left(w_{1}+1\right)+\cdots+\mu_{\ell}\left(w_{\ell}+1\right) .
$$

Recalling the expression of $r(g, \mu)$ given in (2.2) and using (3.7), the Laplace transform of the left-hand side of (3.14) becomes

$$
\begin{aligned}
\sum_{\mu \in \mathbb{N}^{\ell}} r(g, \mu) H_{g}(\mu) \mathrm{e}^{-\langle\mu, w+1\rangle}= & \left(2 g-2+\ell+\sum_{i=1}^{\ell} t_{i}^{2}\left(t_{i}-1\right) \frac{\partial}{\partial t_{i}}\right) \\
& \times \widehat{\mathcal{H}}_{g, \ell}\left(t_{1}, \ldots, t_{\ell}\right) .
\end{aligned}
$$

Here we note that multiplication of $\mu_{i}$ to the summand corresponds to the operation of $D_{i}=t_{i}^{2}\left(t_{i}-1\right) \frac{\partial}{\partial t_{i}}$ due to (4.10).

To find the Laplace transform of the cut terms (3.10), we first note a formula:

$$
\begin{aligned}
& \sum_{\mu_{1}, \mu_{2} \geq 0} f\left(\mu_{1}+\mu_{2}\right) \mathrm{e}^{-\left(\mu_{1} w_{1}+\mu_{2} w_{2}\right)} \\
& =\sum_{k=0}^{\infty} \sum_{m=0}^{k} f(k) \mathrm{e}^{-k w_{1}} \mathrm{e}^{-m\left(w_{2}-w_{1}\right)} \\
& =\sum_{k=0}^{\infty} \frac{1-\mathrm{e}^{-(k+1)\left(w_{2}-w_{1}\right)}}{1-\mathrm{e}^{-\left(w_{2}-w_{1}\right)}} f(k) \mathrm{e}^{-k w_{1}} \\
& =\frac{1}{\mathrm{e}^{-w_{1}}-\mathrm{e}^{-w_{2}}} \sum_{k=0}^{\infty} f(k)\left(\mathrm{e}^{-(k+1) w_{1}}-\mathrm{e}^{-(k+1) w_{2}}\right) .
\end{aligned}
$$

Thus we obtain

$$
\begin{aligned}
& \frac{1}{2} \sum_{\mu \in \mathbb{N}^{\ell}} \sum_{i \neq j}\left(\mu_{i}+\mu_{j}\right) H_{g}\left(\mu(\hat{i}, \hat{j}), \mu_{i}+\mu_{j}\right) \mathrm{e}^{-\langle\mu, w+1\rangle} \\
& \quad=\frac{1}{2} \sum_{i \neq j} \frac{1}{\mathrm{e}^{-\left(w_{i}+1\right)}-\mathrm{e}^{-\left(w_{j}+1\right)}}\left(\mathrm{e}^{-\left(w_{i}+1\right)} t_{i}^{2}\left(t_{i}-1\right) \frac{\partial}{\partial t_{i}} \widehat{\mathcal{H}}_{g, \ell-1}\right.
\end{aligned}
$$




$$
\begin{aligned}
& \left.\times\left(t_{1}, \ldots, \widehat{t_{j}}, \ldots, t_{\ell}\right)-\mathrm{e}^{-\left(w_{j}+1\right)} t_{j}^{2}\left(t_{j}-1\right) \frac{\partial}{\partial t_{j}} \widehat{\mathcal{H}}_{g, \ell-1}\left(t_{1}, \ldots, \widehat{t_{i}}, \ldots, t_{\ell}\right)\right) \\
& -\sum_{i \neq j} t_{i}^{2}\left(t_{i}-1\right) \frac{\partial}{\partial t_{i}} \widehat{\mathcal{H}}_{g, \ell-1}\left(t_{1}, \ldots, \widehat{t_{j}}, \ldots, t_{\ell}\right)
\end{aligned}
$$

where the last term comes from the adjustment of the cases $\mu_{i}=0$ and $\mu_{j}=0$ that are not included in the Laplace transform.

The Laplace transform of the first join terms (3.12) is given by

$$
\begin{aligned}
& \frac{1}{2} \sum_{\mu \in \mathbb{N}^{\ell}} \sum_{i=1}^{\ell} \sum_{\alpha+\beta=\mu_{i}} \alpha \beta H_{g-1}(\mu(\hat{i}), \alpha, \beta) \mathrm{e}^{-\langle\mu, w+1\rangle} \\
& \quad=\sum_{i=1}^{\ell}\left[u_{1}^{2}\left(u_{1}-1\right) u_{2}^{2}\left(u_{2}-1\right) \frac{\partial^{2}}{\partial u_{1} \partial u_{2}} \widehat{\mathcal{H}}_{g-1, \ell+1}\left(u_{1}, u_{2}, t_{L \backslash\{i\}}\right)\right]_{u_{1}=u_{2}=t_{i}}
\end{aligned}
$$

where $t_{I}=\left(t_{i}\right)_{i \in I}$ for a subset $I \subset L=\{1,2, \ldots, \ell\}$. In the same way we can calculate the Laplace transform of the second join terms (3.13):

$$
\begin{aligned}
\sum_{\mu \in \mathbb{N}^{\ell}} & \sum_{\alpha+\beta=\mu_{i}} \alpha \beta \sum_{\substack{g_{1}+g_{2}=g \\
\nu_{1} \sqcup \nu_{2}=\mu(\hat{i})}} H_{g_{1}}\left(\nu_{1}, \alpha\right) H_{g_{2}}\left(\nu_{2}, \beta\right) \mathrm{e}^{-\langle\mu, w+1\rangle} \\
= & {\left[\sum_{\substack{g_{1}+g_{2}=g \\
\sqcup \sqcup K=L \backslash\{i\}}} u_{1}^{2}\left(u_{1}-1\right) \frac{\partial}{\partial u_{1}} \widehat{\mathcal{H}}_{g_{1},|J|+1}\left(u_{1}, t_{J}\right) u_{2}^{2}\left(u_{2}-1\right)\right.} \\
& \left.\times \frac{\partial}{\partial u_{2}} \widehat{\mathcal{H}}_{g_{2},|K|+1}\left(u_{2}, t_{K}\right)\right]_{u_{1}=u_{2}=t_{i}}
\end{aligned}
$$

Thus we establish

$$
\begin{aligned}
& \left(2 g-2+\ell+\sum_{i=1}^{\ell} t_{i}^{2}\left(t_{i}-1\right) \frac{\partial}{\partial t_{i}}\right) \widehat{\mathcal{H}}_{g, \ell}\left(t_{1}, \ldots, t_{\ell}\right) \\
& \quad=\sum_{i<j} \frac{1}{\mathrm{e}^{-\left(w_{i}+1\right)}-\mathrm{e}^{-\left(w_{j}+1\right)}}\left(\mathrm{e}^{-\left(w_{i}+1\right)} t_{i}^{2}\left(t_{i}-1\right) \frac{\partial}{\partial t_{i}} \widehat{\mathcal{H}}_{g, \ell-1}\right.
\end{aligned}
$$




$$
\begin{aligned}
& \left.\times\left(t_{1}, \ldots, \widehat{t_{j}}, \ldots, t_{\ell}\right)-\mathrm{e}^{-\left(w_{j}+1\right)} t_{j}^{2}\left(t_{j}-1\right) \frac{\partial}{\partial t_{j}} \widehat{\mathcal{H}}_{g, \ell-1}\left(t_{1}, \ldots, \widehat{t}_{i}, \ldots, t_{\ell}\right)\right) \\
& -\sum_{i \neq j} t_{i}^{2}\left(t_{i}-1\right) \frac{\partial}{\partial t_{i}} \widehat{\mathcal{H}}_{g, \ell-1}\left(t_{1}, \ldots, \widehat{t}_{j}, \ldots, t_{\ell}\right) \\
& +\sum_{i=1}^{\ell}\left[u_{1}^{2}\left(u_{1}-1\right) u_{2}^{2}\left(u_{2}-1\right) \frac{\partial^{2}}{\partial u_{1} \partial u_{2}} \widehat{\mathcal{H}}_{g-1, \ell+1}\left(u_{1}, u_{2}, t_{L \backslash\{i\}}\right)\right]_{u_{1}=u_{2}=t_{i}} \\
& +\frac{1}{2} \sum_{i=1}^{\ell} \sum_{\substack{g_{1}+g_{2}=g \\
J \sqcup K=L \backslash\{i\}}} t_{i}^{2}\left(t_{i}-1\right) \frac{\partial}{\partial t_{i}} \widehat{\mathcal{H}}_{g_{1},|J|+1}\left(t_{i}, t_{J}\right) \cdot t_{i}^{2}\left(t_{i}-1\right) \\
& \times \frac{\partial}{\partial t_{i}} \widehat{\mathcal{H}}_{g_{2},|K|+1}\left(t_{i}, t_{K}\right) .
\end{aligned}
$$

Note that unstable geometries are contained in the last summation. We use (4.13) and (4.14) to substitute the values in (5.3). The result becomes surprisingly simple due to cancelation of the nonpolynomial terms. For $g_{1}=$ 0 and $J=\emptyset$, the contribution is

$$
\sum_{i=1}^{\ell} \hat{\xi}_{-1}\left(t_{i}\right) t_{i}^{2}\left(t_{i}-1\right) \frac{\partial}{\partial t_{i}} \widehat{\mathcal{H}}_{g, \ell}\left(t_{1}, \ldots, t_{\ell}\right) .
$$

For $g_{1}=0$ and $J=\{j\} \subset L \backslash\{i\}$, we have

$$
\begin{aligned}
t_{i}^{2}\left(t_{i}-1\right) \frac{\partial}{\partial t_{i}} \widehat{\mathcal{H}}_{0,2}\left(t_{i}, t_{j}\right) & =\frac{\hat{\xi}_{0}\left(t_{i}\right)}{\hat{\xi}_{-1}\left(t_{i}\right)-\hat{\xi}_{-1}\left(t_{j}\right)}-\frac{x_{i}}{x_{i}-x_{j}}-\hat{\xi}_{0}\left(t_{i}\right) \\
& =\frac{\hat{\xi}_{0}\left(t_{i}\right)}{\hat{\xi}_{-1}\left(t_{i}\right)-\hat{\xi}_{-1}\left(t_{j}\right)}-\frac{\mathrm{e}^{-\left(w_{i}+1\right)}}{\mathrm{e}^{-\left(w_{i}+1\right)}-\mathrm{e}^{-\left(w_{j}+1\right)}}-\hat{\xi}_{0}\left(t_{i}\right) .
\end{aligned}
$$

Thus the unstable $(0,2)$ contribution in $(5.3)$ is

$$
\begin{aligned}
\sum_{i<j} & \left(\frac{t_{i}^{2}\left(t_{i}-1\right)^{2} \frac{\partial}{\partial t_{i}} \widehat{\mathcal{H}}_{g, \ell-1}\left(t_{1}, \ldots, \widehat{t}_{j}, \ldots, t_{\ell}\right)}{\hat{\xi}_{-1}\left(t_{i}\right)-\hat{\xi}_{-1}\left(t_{j}\right)}\right. \\
& \left.-\frac{t_{j}^{2}\left(t_{j}-1\right)^{2} \frac{\partial}{\partial t_{j}} \widehat{\mathcal{H}}_{g, \ell-1}\left(t_{1}, \ldots, \widehat{t}_{i}, \ldots, t_{\ell}\right)}{\hat{\xi}}\right) \\
& -\sum_{i<j} \frac{1}{\mathrm{e}^{-\left(w_{i}+1\right)}-\mathrm{e}^{-\left(w_{j}+1\right)}}\left(\mathrm{e}^{-\left(w_{i}+1\right)} t_{i}^{2}\left(t_{i}-1\right) \frac{\partial}{\partial t_{i}} \widehat{\mathcal{H}}_{g, \ell-1}\left(t_{1}, \ldots, \widehat{t}_{j}, \ldots, t_{\ell}\right)\right.
\end{aligned}
$$




$$
\begin{aligned}
& \left.-\mathrm{e}^{-\left(w_{j}+1\right)} t_{j}^{2}\left(t_{j}-1\right) \frac{\partial}{\partial t_{j}} \widehat{\mathcal{H}}_{g, \ell-1}\left(t_{1}, \ldots, \widehat{t}_{i}, \ldots, t_{\ell}\right)\right) \\
& -\sum_{i \neq j} \hat{\xi}_{0}\left(t_{i}\right) t_{i}^{2}\left(t_{i}-1\right) \frac{\partial}{\partial t_{i}} \widehat{\mathcal{H}}_{g, \ell-1}\left(t_{1}, \ldots, \widehat{t_{j}}, \ldots, t_{\ell}\right) .
\end{aligned}
$$

We have thus proved the following, which is equivalent to Theorem 1.1.

Theorem 5.1. The Laplace transform of the cut-and-join equation is the following equation for polynomials $\widehat{\mathcal{H}}_{g, \ell}\left(t_{1}, \ldots, t_{\ell}\right)$ subject to the stability condition $2 g-2+\ell>0$ :

$$
\begin{aligned}
& \left(2 g-2+\ell+\sum_{i=1}^{\ell}\left(1-\hat{\xi}_{-1}\left(t_{i}\right)\right) t_{i}^{2}\left(t_{i}-1\right) \frac{\partial}{\partial t_{i}}\right) \widehat{\mathcal{H}}_{g, \ell}\left(t_{1}, \ldots, t_{\ell}\right) \\
& =\sum_{i<j} t_{i} t_{j}\left(\frac{t_{i}^{2}\left(t_{i}-1\right)^{2} \frac{\partial}{\partial t_{i}} \widehat{\mathcal{H}}_{g, \ell-1}\left(t_{1}, \ldots, \widehat{t}_{j}, \ldots, t_{\ell}\right)}{t_{i}-t_{j}}\right. \\
& \left.-\frac{t_{j}^{2}\left(t_{j}-1\right)^{2} \frac{\partial}{\partial t_{j}} \widehat{\mathcal{H}}_{g, \ell-1}\left(t_{1}, \ldots, \widehat{t}_{i}, \ldots, t_{\ell}\right)}{t_{i}-t_{j}}\right) \\
& -\sum_{i \neq j} t_{i}^{3}\left(t_{i}-1\right) \frac{\partial}{\partial t_{i}} \widehat{\mathcal{H}}_{g, \ell-1}\left(t_{1}, \ldots, \widehat{t}_{j}, \ldots, t_{\ell}\right) \\
& +\frac{1}{2} \sum_{i=1}^{\ell}\left[u_{1}^{2}\left(u_{1}-1\right) u_{2}^{2}\left(u_{2}-1\right) \frac{\partial^{2}}{\partial u_{1} \partial u_{2}} \widehat{\mathcal{H}}_{g-1, \ell+1}\left(u_{1}, u_{2}, t_{L \backslash\{i\}}\right)\right]_{u_{1}=u_{2}=t_{i}} \\
& +\frac{1}{2} \sum_{i=1}^{\ell} \sum_{\substack{g_{1}+g_{2}=g \\
J \sqcup K=L \backslash\{i\}}}^{\text {stable }} t_{i}^{2}\left(t_{i}-1\right) \frac{\partial}{\partial t_{i}} \widehat{\mathcal{H}}_{g_{1},|J|+1}\left(t_{i}, t_{J}\right) \cdot t_{i}^{2}\left(t_{i}-1\right) \\
& \times \frac{\partial}{\partial t_{i}} \widehat{\mathcal{H}}_{g_{2},|K|+1}\left(t_{i}, t_{K}\right) \text {. }
\end{aligned}
$$

In the last sum each term is restricted to satisfying the stability conditions $2 g_{1}-1+|J|>0$ and $2 g_{2}-1+|K|>0$.

Remark 5.1. Equation (5.4) is equivalent to the cut-and-join equation (3.4) and (3.14). Many other equivalent formulations have been established, including the differential equation of [16, Theorem 3.1]. 


\section{The Witten-Kontsevich theorem and the $\lambda_{g}$ formula}

It has been noticed that the asymptotic behavior of Hurwitz numbers for a large partition recovers the intersection numbers of $\psi$-classes [32]. Actual recovery of the Witten-Kontsevich theorem $[23,35]$ from the ELSV formula using this asymptotic argument is rather involved ([32], see also [22]). Since the Laplace transform contains all the information of the asymptotics, we can easily deduce the Virasoro constraint equation, or the equivalent DVV formula [6, Equation (4.1)], for the $\psi$-class intersection from our main Equation (1.2). Thus we obtain a straightforward proof of the Witten conjecture. In this section we observe that the top degree terms of the recursion is the DVV formula. We also examine that the lowest degree terms imply the descendant relation of the $\lambda_{g}$ formula [11,12]. Our argument is along the same line with $[4,16,20]$. However, due to the polynomial formulation of (1.2), the derivation becomes simpler.

First we compute the polynomial $\hat{\xi}_{n}(t)$ using (4.11). It has the general form

$$
\hat{\xi}_{n}(t)=(2 n-1) ! ! t^{2 n+1}-\frac{(2 n+1) ! !}{3} t^{2 n}+\cdots+a_{n} t^{n+2}+(-1)^{n} n ! t^{n+1},
$$

where $a_{n}$ is defined by

$$
a_{n}=-\left[(n+1) a_{n-1}+(-1)^{n} n !\right]
$$

and is identified as the sequence A001705 or A081047 of the On-Line Encyclopedia of Integer Sequences.

The DVV formula for the Virasoro constraint condition on the $\psi$-class intersections reads

$$
\begin{aligned}
\left\langle\tau_{n_{L}}\right\rangle_{g, \ell}= & \sum_{j \geq 2} \frac{\left(2 n_{1}+2 n_{j}-1\right) ! !}{\left(2 n_{1}+1\right) ! !\left(2 n_{j}-1\right) ! !}\left\langle\tau_{n_{1}+n_{j}-1} \tau_{n_{L \backslash\{1, j\}}}\right\rangle_{g, \ell-1}+\frac{1}{2} \sum_{a+b=n_{1}-2} \\
& \times\left(\left\langle\tau_{a} \tau_{b} \tau_{n_{L \backslash\{1\}}}\right\rangle_{g-1, \ell+1}+\sum_{\substack{g_{1}+g_{2}=g \\
J \sqcup K=L \backslash\{1\}}}^{\text {stable }}\left\langle\tau_{a} \tau_{n_{J}}\right\rangle_{g_{1},|J|+1} \cdot\left\langle\tau_{b} \tau_{n_{K}}\right\rangle_{g_{2},|K|+1}\right) \\
& \times \frac{(2 a+1) ! !(2 b+1) ! !}{\left(2 n_{1}+1\right) ! !} .
\end{aligned}
$$


Here $L=\{1, \ldots, \ell\}$ is the index set as before, and for a subset $I \subset L$ we write

$$
n_{I}=\left(n_{i}\right)_{i \in I} \quad \text { and } \quad \tau_{n_{I}}=\prod_{i \in I} \tau_{n_{i}}
$$

Proposition 6.1. The $D V V$ formula (6.2) is exactly the relation among the top degree coefficients of recursion (1.2).

Proof. Choose $n_{L}$ so that $\left|n_{L}\right|=n_{1}+n_{2}+\cdots+n_{\ell}=3 g-3+\ell$. The degree of the left-hand side of $(1.2)$ is $3(2 g-2+\ell)+1$. So we compare the coefficients of $t_{1}^{2 n_{1}+2} \prod_{j \geq 2} t_{j}^{2 n_{j}+1}$ in the recursion formula. The contribution from the left-hand side of $(1.2)$ is

$$
\left\langle\tau_{n_{L}}\right\rangle_{g, \ell}\left(2 n_{1}+1\right) ! ! \prod_{j \geq 2}\left(2 n_{j}-1\right) ! !
$$

The contribution from the first line of the right-hand side comes from

$$
\begin{aligned}
\sum_{j \geq 2}\left\langle\tau_{m} \tau_{n_{L \backslash\{1, j\}}}\right\rangle_{g, \ell-1}(2 m+1) ! ! & t_{1}^{2} t_{j} t_{1}^{2 m+3}-t_{j}^{2} t_{1} t_{j}^{2 m+3} \\
t_{1}-t_{j} & \sum_{j \geq 2}\left\langle\tau_{m} \tau_{n_{L \backslash\{1, j\}}}\right\rangle_{g, \ell-1}(2 m+1) ! ! t_{1} t_{j} \frac{t_{1}^{2 m+4}-t_{j}^{2 m+4}}{t_{1}-t_{j}} \\
= & \sum_{j \geq 2}\left\langle\tau_{m} \tau_{n_{L \backslash\{1, j\}}}\right\rangle_{g, \ell-1}(2 m+1) ! ! \sum_{a+b=2 m+3} t_{1}^{a+1} t_{j}^{b+1},
\end{aligned}
$$

where $m=n_{1}+n_{j}-1$. The matching term in this formula is $a=2 n_{1}+1$ and $b=2 n_{j}$. Thus we extract as the coefficient of $t_{1}^{2 n_{1}+2} \prod_{j \geq 2} t_{j}^{2 n_{j}+1}$

$$
\sum_{j \geq 2}\left\langle\tau_{n_{1}+n_{j}-1} \tau_{n_{L \backslash\{1, j\}}}\right\rangle_{g, \ell-1}\left(2 n_{1}+2 n_{j}-1\right) ! ! \prod_{k \neq 1, j}\left(2 n_{k}-1\right) ! ! .
$$

The contributions of the second and the third lines of the right-hand side of (1.2) are

$$
\begin{aligned}
& \frac{1}{2} \sum_{a+b=n_{1}-2}\left(\left\langle\tau_{a} \tau_{b} \tau_{L \backslash\{1\}}\right\rangle_{g-1, \ell+1}+\frac{1}{2} \sum_{\substack{g_{1}+g_{2}=g \\
J \sqcup K=L \backslash\{1\}}}^{\text {stable }}\left\langle\tau_{a} \tau_{n_{J}}\right\rangle_{g_{1},|J|+1}\left\langle\tau_{b} \tau_{n_{K}}\right\rangle_{g_{2},|K|+1}\right) \\
& \quad \times(2 a+1) ! !(2 b+1) ! ! \prod_{j \geq 2}\left(2 n_{j}-1\right) ! !
\end{aligned}
$$


We have thus recovered the Witten-Kontsevich theorem [6, 23, 35].

The $\lambda_{g}$ formula $[11,12,25,26]$ is

$$
\left\langle\tau_{n_{L}} \lambda_{g}\right\rangle_{g, \ell}=\left(\begin{array}{c}
2 g-3+\ell \\
n_{L}
\end{array}\right) b_{g}
$$

where

$$
\left(\begin{array}{c}
2 g-3+\ell \\
n_{L}
\end{array}\right)=\left(\begin{array}{c}
2 g-3+\ell \\
n_{1}, \ldots, n_{\ell}
\end{array}\right)
$$

is the multinomial coefficient, and

$$
b_{g}=\frac{2^{2 g-1}-1}{2^{2 g-1}} \frac{\left|B_{2 g}\right|}{(2 g) !}
$$

is a coefficient of the series

$$
\sum_{j=0}^{\infty} b_{j} s^{2 j}=\frac{s / 2}{\sin (s / 2)} .
$$

Proposition 6.2. The lowest degree terms of the topological recursion (1.2) proves the combinatorial factor of the $\lambda_{g}$ formula

$$
\left\langle\tau_{n_{L}} \lambda_{g}\right\rangle_{g, \ell}=\left(\begin{array}{c}
2 g-3+\ell \\
n_{L}
\end{array}\right)\left\langle\tau_{2 g-1} \lambda_{g}\right\rangle_{g, 1} .
$$

Proof. Choose $n_{L}$ subject to $\left|n_{L}\right|=2 g-3+\ell$. We compare the coefficient of the terms of $\prod_{i \geq 1} t_{i}^{n_{i}+1}$ in (1.2), which has degree $\left|n_{L}\right|+\ell=2 g-3+2 \ell$. The left-hand side contributes

$$
\begin{aligned}
& (-1)^{2 g-3+\ell}(-1)^{g}\left\langle\tau_{n_{L}} \lambda_{g}\right\rangle_{g, \ell} \prod_{i \geq 1} n_{i} !\left(2 g-2+\ell-\sum_{i=1}^{\ell}\left(n_{i}+1\right)\right) \\
& =(-1)^{\ell}(-1)^{g}\left\langle\tau_{n_{L}} \lambda_{g}\right\rangle_{g, \ell}(\ell-1) \prod_{i \geq 1} n_{i} ! .
\end{aligned}
$$

The lowest degree terms of the first line of the right-hand side are

$$
\begin{aligned}
& (-1)^{g} \sum_{i<j} \sum_{m}\left\langle\tau_{m} \tau_{L \backslash\{i, j\}} \lambda_{g}\right\rangle_{g, \ell-1}(-1)^{m}(m+1) ! \\
& \times \frac{t_{i}^{m+4}-t_{j}^{m+4}}{t_{i}-t_{j}}(-1)^{2 g-3+\ell-n_{i}-n_{j}} \prod_{k \neq i, j} n_{k} ! t_{k}^{n_{k}+1} .
\end{aligned}
$$


Since $m=n_{i}+n_{j}-1$, the coefficient of $\prod_{i \geq 1} t_{i}^{n_{i}+1}$ is

$$
-(-1)^{g}(-1)^{2 g-3+\ell} \sum_{i<j}\left\langle\tau_{n_{i}+n_{j}-1} \tau_{L \backslash\{i, j\}} \lambda_{g}\right\rangle_{g, \ell-1}\left(\begin{array}{c}
n_{i}+n_{j} \\
n_{i}
\end{array}\right) \prod_{i \geq 1} n_{i} ! .
$$

Note that the lowest degree coming from the second and the third lines of the right-hand side of (1.2) is $\left|n_{L}\right|+\ell+2$, which is higher than the lowest degree of the left-hand side. Therefore, we have obtained a recursion equation with respect to $\ell$

$$
(\ell-1)\left\langle\tau_{n_{L}} \lambda_{g}\right\rangle_{g, \ell}=\sum_{i<j}\left\langle\tau_{n_{i}+n_{j}-1} \tau_{L \backslash\{i, j\}} \lambda_{g}\right\rangle_{g, \ell-1}\left(\begin{array}{c}
n_{i}+n_{j} \\
n_{i}
\end{array}\right) .
$$

The solution of the recursion equation (6.6) is the multinomial coefficient (6.4).

Remark 6.1. Although the topological recursion (1.2) determines all linear Hodge integrals, the closed formula

$$
b_{g}=\left\langle\tau_{2 g-2} \lambda_{g}\right\rangle_{g, 1} \quad g \geq 1
$$

does not seem to follow directly from it.

\section{Acknowledgments}

The authors thank the American Institute of Mathematics for the hospitality during their stay that promoted this collaboration. They are grateful to Ravi Vakil, Lin Chen, and the referee for useful comments. M.M. thanks Herbert Kurke for giving him the opportunity to lecture on Hurwitz numbers based on [32,33] at Humboldt Universität zu Berlin in 2002 and 2005. M.M. also thanks the NSF, Kyoto University, the Institute for the Physics and Mathematics of the Universe in Tokyo, the Osaka City University Advanced Mathematical Institute, Tôhoku University, KIAS in Seoul, and the University of Salamanca for their hospitality and financial support during the preparation of this work. Dedicated to Herbert Kurke on the occasion of his 70th birthday. 


\section{References}

[1] G. Borot, B. Eynard, M. Mulase and B. Safnuk, Hurwitz numbers, matrix models and topological recursion, 2009, arXiv:0906.1206 [math. $\mathrm{Ph}]$.

[2] V. Bouchard, A. Klemm, M. Mariño and S. Pasquetti, Remodeling the B-model, Commun. Math. Phys. 287 (2008), 117-178.

[3] V. Bouchard and M. Mariño, Hurwitz numbers, matrix models and enumerative geometry, Proc. Symposia Pure Math. 78 (2008), 263-283.

[4] L. Chen, Y. Li and K. Liu, Localization, Hurwitz numbers and the Witten conjecture, 2006, arXiv:math.AG/0609263 [math.AG].

[5] R. Dijkgraaf, Intersection theory, integrable hierarchies and topological field theory, New Symmetry Principles in Quantum Field Theory (Cargése, 1991), NATO Adv. Sci. Inst. Ser. B Phys., 295, Plenum, New York, 1992, 95-158.

[6] R. Dijkgraaf, E. Verlinde and H. Verlinde, Loop equations and Virasoro constraints in non-perturbative two-dimensional quantum gravity, Nucl. Phys. B348 (1991), 435-456.

[7] T. Ekedahl, S. Lando, M. Shapiro and A. Vainshtein, Hurwitz numbers and intersections on moduli spaces of curves, Invent. Math. 146 (2001), $297-327$.

[8] B. Eynard, M. Mulase and B. Safnuk, The Laplace transform of the cut-and-join equation and the Bouchard-Mariño conjecture on Hurwitz numbers, 2009, arXiv:0907.5224 [math.AG].

[9] B. Eynard and N. Orantin, Invariants of algebraic curves and topological expansion, Commun. Number Theory Phys. 1 (2007), $347-452$.

[10] B. Eynard and N. Orantin, Weil-Petersson volume of moduli spaces, Mirzakhani's recursion and matrix models, 2007, arXiv:0705.3600 [math-ph].

[11] C. Faber and R. Pandharipande, Hodge integrals and Gromov-Witten theory, Invent. Math. 139 (2000), 173-199.

[12] C. Faber and R. Pandharipande, Hodge integrals, partition matrices, and the $\lambda_{g}$ conjecture, Ann. of Math. 157 (2003), 97-124. 
[13] I.P. Goulden and D.M. Jackson, Transitive factorisations into transpositions and holomorphic mappings on the sphere, Proc. A.M.S., 125 (1997), 51-60.

[14] I.P. Goulden, D.M. Jackson and A. Vainshtein, The number of ramified coverings of the sphere by the torus and surfaces of higher genera, Ann. of Comb. 4 (2000), 27-46.

[15] I.P. Goulden, D.M. Jackson and R. Vakil, The Gromov-Witten potential of a point, Hurwitz numbers, and Hodge integrals, Proc. London Math. Soc. 83(3) (2001), 563-581.

[16] I.P. Goulden, D.M. Jackson and R. Vakil, A short proof of the $\lambda_{g}$ conjecture without Gromov-Witten theory: Hurwitz theory and the moduli of curves, 2006, arXiv:math/0604297v1 [math.AG].

[17] T. Graber and R. Vakil, Hodge integrals and Hurwitz numbers via virtual localization, Compositio Math. 135 (2003), 25-36.

[18] T. Graber and R. Vakil, Relative virtual localization and vanishing of tautological classes on moduli spaces of curves, Duke Math. J. 130 (2005), 1-37.

[19] A. Hurwitz, Über Riemann'sche Flächen mit gegebene Verzweigungspunkten, Math. Ann. 39 (1891), 1-66.

[20] M. Kazarian, KP hierarchy for Hodge integrals, 2008, arXiv:0809.3263.

[21] M. Kazarian and S. Lando, An algebro-geometric proof of Witten's conjecture, J. Amer. Math. Soc. 20 (2007), 1079-1089.

[22] Y.S. Kim and K. Liu, A simple proof of Witten conjecture through localization, 2005, Preprint, arXiv:math/0508384 [math.AG].

[23] M. Kontsevich, Intersection theory on the moduli space of curves and the matrix Airy function, Commun. Math. Phys. 147 (1992), 1-23.

[24] A.M. Li, G. Zhao, and Q. Zheng, The number of ramified coverings of a Riemann surface by Riemann surface, Commun. Math. Phys. 213 (2000), 685-696.

[25] C.-C. M. Liu, Formulae of one-partition and two-partition Hodge integrals, Geom. Top. Monographs 8 (2006), 105-128. 
[26] C.-C. M. Liu, K. Liu and J. Zhou, A proof of a conjecture of MariñoVafa on Hodge integrals, J. Differential Geom. 65(2) (2003), 289-340.

[27] K. Liu and H. Xu, A simple proof of Mirzakhani's recursion formula of Weil-Petersson volumes, arXiv:0705.2086 [math.AG].

[28] K. Liu and H. Xu, Recursion formulae of higher Weil-Petersson volumes, Intern. Math. Res. Notices, 2009(5) (2009), 835-859.

[29] M. Mirzakhani, Simple geodesics and Weil-Petersson volumes of moduli spaces of bordered Riemann surfaces, Invent. Math. 167 (2007), 179222 .

[30] M. Mirzakhani, Weil-Petersson volumes and intersection theory on the moduli space of curves, J. Amer. Math. Soc. 20 (2007), 1-23.

[31] M. Mulase and B. Safnuk, Mirzakhani's recursion relations, Virasoro constraints and the KdV hierarchy, Indian J. Math. 50 (2008), 189-228.

[32] A. Okounkov and R. Pandharipande, Gromov-Witten theory, Hurwitz numbers, and matrix models, I, Proc. Symposia Pure Math. 80 (2009), 325-414.

[33] A. Okounkov and R. Pandharipande, The equivariant Gromov-Witten theory of $\mathbb{P}^{1}, 2002$, arXiv:math/0207233 [math.AG].

[34] R. Vakil, Harvard thesis 1997.

[35] E. Witten, Two dimensional gravity and intersection theory on moduli space, Surveys Differential Geom. 1 (1991), 243-310.

[36] J. Zhou, Hodge integrals, Hurwitz numbers, and symmetric groups, 2003, Preprint, arXiv:math/0308024 [math.AG].

Department of Mathematics

UNIVERSITY OF CALIFORNIA

DAVIS, CA 95616-8633,

USA

E-mail addresses: nzhzhang@math.ucdavis.edu, mulase@math.ucdavis.edu

Received August 19, 2009 
